\title{
AN ELEMENTARY PROOF OF A RADON-NIKODÝM THEOREM FOR FINITELY ADDITIVE SET FUNCTIONS
}

\author{
JAN PACHL
}

\begin{abstract}
In 1967 Charles Fefferman proved a RadonNikodým theorem for finitely additive measures. We give an elementary proof of a generalization of this theorem.
\end{abstract}

In this paper we give an elementary proof of the

THEOREM. Let $\mu, \gamma$ be real-valued bounded finitely additive set functions on an algebra $\Sigma$ of subsets of the set $S$. Then for every $\varepsilon>0$ there exists $N \in \Sigma$ and a $\mu$-simple function $f$ on $S$ such that

$$
\begin{aligned}
E \in \Sigma \& E \subseteq N & \Rightarrow|\mu E|<\varepsilon, \\
E \in \Sigma \& E \subseteq S \backslash N & \Rightarrow\left|\gamma E-\int_{E} f d \mu\right|<\varepsilon .
\end{aligned}
$$

(Hence there is a $\mu$-simple function g with $E \in \Sigma \Rightarrow\left|\gamma E-\gamma(E \cap N)-\int_{E} g d \mu\right|<\varepsilon$.)

If $\gamma$ is absolutely $\mu$-continuous (i.e., for every $\varepsilon>0$ there exists $\delta>0$ such that $|\mu E|<\delta \Rightarrow|\gamma E|<\varepsilon$ ), the Theorem reduces to a result of Fefferman which he proved using functional analysis (see Corollary).

LEMMA 1 ("WEAK HAHN DECOMPOSITION"). Let $v$ be a real-valued bounded finitely additive set function on an algebra $\Sigma \subseteq \exp S$. For every $\alpha>0$ there is a $B \in \Sigma($ written $B(\nu, \alpha))$ such that

$$
E \in \Sigma \& E \subseteq B \Rightarrow \nu E>-\alpha, \quad E \in \Sigma \& E \subseteq S \backslash B \Rightarrow \nu E<\alpha .
$$

Proof. $\quad v$ is bounded and so there is a smallest natural number $k$ with $\nu \leqq k \alpha$; for some $B \in \Sigma$ we have $\nu B>k \alpha-\alpha$. If $E \in \Sigma$ and $E \subseteq B$, then $v E=v B-v(B \backslash E)>k \alpha-\alpha-k \alpha=-\alpha$; if $E \in \Sigma$ and $E \subseteq S \backslash B$, then $v E=$ $v(B \cup E)-v B<k \alpha-(k \alpha-\alpha)=\alpha$.

Lemma 2 (CF. [2, §22.2], [1, Theorem 2]). Let $\mu, \gamma$ be finite realvalued finitely additive set functions on an algebra $\Sigma \subseteq \exp S$. If there exists $\delta>0$ such that $\mu>-\delta / 2$ and $\gamma>-\delta / 2$, then there exist $N \in \Sigma$ and $a \mu$ simple function $f$ on $S$ such that

Received by the editors August 27, 1970.

AMS 1970 subject classifications. Primary 28-00, 28A25, 28A80.

(c) American Mathematical Society 1972 


$$
\begin{aligned}
E \in \Sigma \& E \subseteq N \Rightarrow|\mu E|<\delta, \\
E \in \Sigma \& E \subseteq S \backslash N \Rightarrow\left|\gamma E-\int_{E} f d \mu\right|<\delta .
\end{aligned}
$$

Proof. Let $m$ be an integer $>2(\gamma S+\delta) \cdot(\mu S+\delta) / \delta^{2}$. Put

$$
\begin{aligned}
B_{k} & \left.=B\left(\gamma-\frac{\delta k}{2(\mu S+\delta)} \mu, \frac{\delta}{2 m}\right) \quad \text { (by Lemma } 1\right), \\
A_{1} & =S \backslash \bigcup_{j=1}^{m} B_{j}, \quad A_{k}=B_{k-1} \backslash \bigcup_{j=k}^{m} B_{j}, \quad k=2,3, \cdots, m, \\
N & =B_{m}, \quad f=\sum_{k=1}^{m} \frac{\delta(k-1)}{2(\mu S+\delta)} \chi_{A_{k}},
\end{aligned}
$$

where $\chi_{A}$ is the characteristic function of $A$. If $E \in \Sigma$ and $E \subseteq N$, then

thus

$$
\gamma E-\frac{\delta m}{2(\mu S+\delta)} \mu E>-\frac{\delta}{2 m} \geqq-\frac{\delta}{2} ;
$$

$$
\begin{aligned}
\mu E< & \frac{2}{m} \frac{\mu S+\delta}{\delta}\left(\gamma E+\frac{\delta}{2}\right)<\frac{\delta^{2}}{(\gamma S+\delta)(\mu S+\delta)} \frac{\mu S+\delta}{\delta} \\
& \times\left(\gamma S-\gamma(S \mid E)+\frac{\delta}{2}\right)<\frac{\delta}{\gamma S+\delta}\left(\gamma S+\frac{\delta}{2}+\frac{\delta}{2}\right)=\delta .
\end{aligned}
$$

If $E \in \Sigma$ and $E \subseteq S \backslash N$, then $E$ is the disjoint union of the sets $E_{1}, \cdots, E_{m}$, where $E_{k}=E \cap A_{k}$ for $k=1,2, \cdots, m$. Moreover, since $E_{k} \subseteq A_{k} \subseteq S \backslash B_{k}$, we have

$$
\gamma E_{k}-\frac{\delta k}{2(\mu S+\delta)} \mu E_{k}<\frac{\delta}{2 m} \text { for } k=1,2, \cdots, m,
$$

and since $E_{k} \subseteq A_{k} \subseteq B_{k-1}$, we have

Hence

$$
\gamma E_{k}-\frac{\delta(k-1)}{2(\mu S+\delta)} \mu E_{k}>-\frac{\delta}{2 m} \text { for } k=2, \cdots, m .
$$

$$
\begin{aligned}
-\delta<-\frac{\delta}{2}+(m-1) & \left(-\frac{\delta}{2 m}\right)<\sum_{k=1}^{m}\left(\gamma E_{k}-\frac{\delta(k-1)}{2(\mu S+\delta)} \mu E_{k}\right) \\
& =\sum_{k=1}^{m}\left(\gamma E_{k}-\frac{\delta k}{2(\mu S+\delta)} \mu E_{k}\right)+\sum_{k=1}^{m} \frac{\delta}{2(\mu S+\delta)} \mu E_{k} \\
& <m \frac{\delta}{2 m}+\frac{\delta}{2} \frac{\mu E}{\mu S+\delta}<\delta .
\end{aligned}
$$


Since $\sum_{k=1}^{m}\left(\gamma E_{k}-(\delta(k-1) / 2(\mu S+\delta)) \mu E_{k}\right)=\gamma E-\int_{E} f d \mu$, Lemma 2 is proved.

Proof of The TheOrem. Let $B=B(\mu, \varepsilon / 8), B^{\prime}=B(\gamma, \varepsilon / 8), \mu_{1} E=$ $\mu(E \cap B), \mu_{2} E=-\mu(E \backslash B), \gamma_{1} E=\gamma\left(E \cap B^{\prime}\right)$ and $\gamma_{2} E=-\gamma\left(E \backslash B^{\prime}\right)$ for $E \in \Sigma$ (so that $\mu=\mu_{1}-\mu_{2}$ and $\gamma=\gamma_{1}-\gamma_{2}$ ). Clearly $\mu_{i}>-\varepsilon / 8$ and $\gamma_{i}>\varepsilon / 8$ for $i=1,2$. By Lemma 2 (with $\delta=\varepsilon / 4$ ) for $i, j=1,2$ there are sets $N_{i j}$ and $\mu$ simple functions $f_{i: i}$ such that

$$
\begin{gathered}
E \in \Sigma \& E \subseteq N_{i j} \Rightarrow\left|\mu_{i} E\right|<\frac{\varepsilon}{4}, \\
E \in \Sigma \& E \subseteq S \backslash N_{i j} \Rightarrow\left|\gamma_{j} E-\int_{E} f_{i j} d \mu_{i}\right|<\frac{\varepsilon}{4} .
\end{gathered}
$$

Let $N=\bigcup_{i, j=1,2} N_{i j}$ and $f=\left(f_{11}-f_{12}\right) \chi_{B}+\left(f_{21}-f_{22}\right) \chi_{S \backslash B}$. If $E \in \Sigma$ and $E \subseteq N$, then $E$ is the disjoint union of sets $E_{i j}, i, j=1,2$, with $E_{i j} \in \Sigma$ and $E_{i j} \subseteq N_{i j}$,

$$
\mu E=\sum_{i, j=1,2} \mu E_{i j} \text { and }-\varepsilon<4 \cdot\left(-\frac{\varepsilon}{8}\right)<\sum_{i, j=1,2} \mu E_{i j}<4 \cdot \frac{\varepsilon}{4}=\varepsilon,
$$

i.e., $|\mu E|<\varepsilon$. If $E \in \Sigma$ and $E \subseteq S \backslash N$, then $E \subseteq S \backslash N_{i j}$ for $i, j=1,2$; in addition $\mu_{2}(E \cap B)=\mu_{1}(E \backslash B)=0$, and thus

$$
\begin{aligned}
\left|\gamma E-\int_{E} f d \mu\right| \leqq & \gamma(E \cap B)-\int_{E \cap B}\left(f_{11}-f_{12}\right) d \mu \mid \\
& +\left|\gamma(E \backslash B)-\int_{E \backslash B}\left(f_{21}-f_{22}\right) d \mu\right| \\
\leqq & \left|\gamma_{1}(E \cap B)-\int_{E \cap B} f_{11} d \mu_{1}\right| \\
& +\left|\gamma_{2}(E \cap B)-\int_{E \cap B} f_{12} d \mu_{1}\right| \\
& +\left|\gamma_{1}(E \mid B)-\int_{E \backslash B} f_{21} d \mu_{2}\right| \\
& +\left|\gamma_{2}(E \mid B)-\int_{E \backslash B} f_{22} d \mu_{2}\right|<4 \cdot \frac{\varepsilon}{4}=\varepsilon .
\end{aligned}
$$

The Theorem is proved.

It is now easy to prove the

COROLlaRY. Let $\mu, \gamma$ be bounded real-valued finitely additive set functions on an algebra $\Sigma \subseteq \exp S$. Let $\gamma$ be absolutely $\mu$-continuous (in the 
sense mentioned above). Then for every $\varepsilon>0$ there is a $\mu$-simple function $f$ such that

$$
E \in \Sigma \Rightarrow\left|\gamma E-\int_{E} f d \mu\right|<\varepsilon .
$$

As Fefferman [1, Lemma 1] has shown, it suffices to suppose that $\gamma$ is finite (instead of bounded).

By the Corollary (if $\mu$ and $\gamma$ satisfy its hypotheses), there is a sequence $\left\{f_{n}\right\}$ of $\mu$-simple functions $f_{n}$ with $\lim _{n \rightarrow \infty} \int_{E} f_{n} d \mu=\gamma E$ uniformly for $E \in \Sigma$ (it was in this form formulated in [1]).

The following example shows there need not be an increasing such sequence:

$S$ is the set of all natural numbers $(\geqq 1)$,

$\Sigma$ is the algebra of all finite subsets of $S$ and their complements,

$$
\begin{array}{lll}
\gamma E=0, & \mu E=\sum_{k \in E} 2^{-k} & \text { for finite } E, \\
\gamma E=1, & \mu E=1+\sum_{k \in E} 2^{-k} & \text { for infinite } E .
\end{array}
$$

\section{REFERENCES}

1. Charles Fefferman, A Radon-Nikodým theorem for finitely additive set functions, Pacific J. Math. 23 (1967), 35-45. MR 35 \#6791.

2. Eduard Cech, Point sets, Jednota Ceskoslov. Mat. a Fyz., Prague, 1936. (Czech).

Department of Mathematics, Charles University, Prague, Czechoslovakia 